\title{
INTERNAL PRECISION IN CCD ASTROMETRY OF QSO OPTICAL COUNTERPARTS
}

\author{
G. Chiumiento(1), L. Lanteri ${ }^{(1)}$, M.G. Lattanzi ${ }^{(2,1)}$, G. Massone $\mathrm{e}^{(1)}$ \\ (1) Torino Astronomical Observatory, 10025 Pino Torinese (TO), Italy \\ (2) Space Telescope Science Institute, Baltimore MD 21218, USA \\ Affiliated with the Astrophysics Division, SSD, ESA
}

\begin{abstract}
The main purpose of this contribution is twofold. First, we report on a preliminary assessment of the astrometric properties of the CCD system we are presently using to extend the magnitude limit of the Torino program on optical positions of QSO's. This assessment is based on the internal consistency of the positions derived from different CCD frames of the same objects. Then, we give a first evaluation of the precision of CCD derived QSO positions after extending a plate based reference frame (secondary frame) onto the CCD images (tertiary frame).

Special emphasis is given to comparisons with plate positions of the same faint objects. It is shown that use of the CCD camera promises a factor of five (or larger) improvement in the precision of QSO positions.
\end{abstract}

\section{Introduction}

The extention to fainter magnitudes $(V>16.5)$ of the Torino program on optical positions of extragalactic radio-sources (Chiumiento et al. 1987, Chiumiento et al. 1989) brought us to experiment with the 320 × 512 pixels RCA CCD (1 square pixel $=30 \mu \mathrm{m}$ on a side) attached to the $1.5 \mathrm{~m}$ Ritchey-Chretien of the University of Bologna. The CCD system operated at a temperature of about $120^{\circ} \mathrm{K}$ and the telescope scale at the CCD chip is $0.51 \%$ /pixel.

Several targets exist in our observing list such that 3 or more stars (besides the QSO) are measurable on our photographic plates within the CCD field $\left(2^{\prime} .7 \times 4^{\prime} .4\right)$. The plates are calibrated via CAMC stars (La Palma 1989); thus the CCD frames can be tied to the CAMC (FK5) system by using the stars surrounding the QSO as the link stars.

In the next section we will briefly describe the available CCD observations. Then, we report on the internal precision of our CCD astrometry. Finally, a preliminary evaluation of the precision of CCD based QSO positions is given in section 5. There, we also compare the quality of our CCD astrometry to photographic errors we had previously obtained for the same targets. 


\section{Observations}

During four nights (Jan 24, Feb 5-7, 1990) partially devoted to this program, we obtained 34 CCD frames, B and V colors, of 10 radiosources of our main program. The exposure times ranged from 5 to 30 minutes. The February nights were during full Moon; thus about one third of the available exposures have an abnormally high sky background. The number of usable link stars in the frames varies from 3 to 10 .

\section{Reduction of the CCD frames}

As usual, the CCD frames are first corrected for dark current, bias, and pixel-topixel sensitivity variations (flat fielding). Then, the astrometric image processing is done using the PC-based software ROBIN developed at Torino Observatory (Lanteri 1990). ROBIN is a package specifically designed for astrometric reductions of both CCD and PDS images.

ROBIN fits a bidimensional gaussian-like function to the star images, plus a linear (both in $\mathrm{x}$ and $\mathrm{y}$ coordinates) polynomial to take into account the sky background. The fits are performed on windows extracted from the frames, whose sizes vary with object magnitudes.

ROBIN outputs the estimated $x$ and $y$ coodinates (in pixel units) of the objects successfully centered (i.e. the center of the bidimensional gaussian model) and their dispersions $(\sigma$ 's). Instrumental magnitudes of the same objects are also derived.

Finally, these quantities are processed through our standard astrometric software.

\section{Internal precision of CCD Astrometry}

For this preliminary study of the internal precision of CCD based positions, we used frames of comparable exposure times on the two QSO's 1148-001 and $1611+343$ (Argue et al., 1984). Both targets have a B magnitude close to $17^{m} .5$. Ten and seven star-like images (besides the QSO's) were successfully centroided (see sec. III) in the fields of $1148-001$ and $1611+343$ respectively. The signal-to-noise ratios of the different images varied from $S / N \simeq 25$ (for objects of $B \simeq 16^{m}$ ) to $S / N \simeq 3$ (for $B \simeq 20^{m}$ ).

Also, through the estimated $\sigma$ 's of the stars, we computed the FWHM of the PSF of each frame $(F W H M \simeq 2.4 \times \sigma)$. A typical $\sigma$ during our observing nights was 1.6 pixel, which gives a typical seeing of $\simeq 2$ ".

The internal astrometric error was determined by comparison of the different exposures after bringing each of them into the same instrumental reference frame via a 6 -constant least squares adjustment.

The average error in one coordinate ranges from 0.02 pixel $(\simeq 0$ ".01) for the "bright" objects $\left(\langle B\rangle \simeq 17^{m}\right)$ to about 0.15 pixel $(\simeq 0$ ".07) for those close to the frame limit $\left(\angle B>\simeq 20^{m}\right)$.

Estimates of the photometric internal precision were calculated as well. The average errors vary from $0^{m} .01$ for the bright objects to $0^{m} .15$ for the faint ones. 


\section{CCD based QSO positions}

In order to evaluate the internal consistency of QSO positions, we selected test objects from our main list for which we have both plate and CCD series. Unfortunately, the CCD run covered a range in right ascension poorly covered by our reduced photographic material. Anyway, we had available 2 plates of $1611+343$. Both plates were taken at the same telescope used for the CCD observations. One of the two plates covers a square field of about $65^{\prime}$ on a side and contains 13 CAMC stars. On this plate we measured both the secondary (15 stars) and the tertiary (7 stars) reference frames. The secondary link stars were used to reduce the other plate which is only $9 \times 12 \mathrm{~cm}$. The magnitudes of the CAMC stars range from $\mathrm{V}=10$ to $\mathrm{V}=13$. Secondary link stars have magnitudes between $14^{\mathrm{m}}$ and $16^{\mathrm{m}}$ and the Tertiary stars cover the interval from $16.5^{\mathrm{m}}$ to $17.5^{\mathrm{m}}$.

Given the photographic tertiary frame, the three CCD frames available for $1611+343$ were independently reduced. Then, the three positions were intercompared to evaluate the internal consistency. The resulting root-mean-square errors are $\pm 0 " .038$ in RA and $\pm 0 " .047$ in DEC. This result, although very preliminary, points to an improvement of about a factor of 10 over the traditional photographic technique at $B \simeq 17^{m} .5$, as shown in Figure 1 .

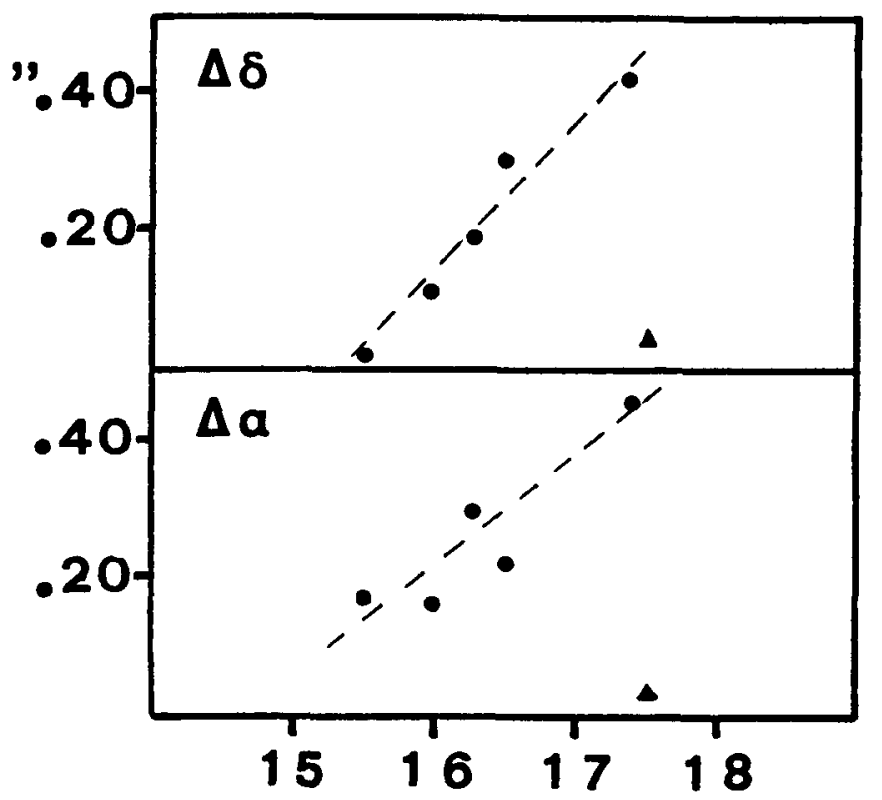

Figure 1. Photographic vs CCD astrometric precision

$$
\bullet=\text { Photographic; } \Delta=\mathrm{CCD}
$$




\section{REFERENCES}

Argue, A.N., De Vegt, C., Elsmore, B. et al.: 1984, Astron.Astrophys., 130, 191. Carlsberg Meridian Catalogue No. 4, La Palma 1989. Eds. Copenhagen Univ. Observatory, Royal Greenwich Observatory, Instituto y Obs. de San Fernando. Chiumiento, G., Lattanzi, M.G., Massone, G., Morbidelli, R., Pannunzio, R., Sarasso, M.: in "Fundamentals of Astrometry", IAU Coll. No. 100, Belgrade 1987, Ed. H.K. Eichhorn, Astrophys. Space Science, in press.

Chiumiento, G., Lattanzi, M.G., Massone, G., Morbidelli, R., Pannunzio, R., Sarasso, M.: 1990, Inertial Coordinate Systems on the Sky, Leningrad 1989, 501-502, Eds. J.H. Lieske and V.K. Abalakin, Kluwer Acad. Publ., Dordrecht. Lanteri, L.: 1990, Osserv. Astron. di Torino, Internal Report no. 16. 